\title{
TOF-SIMS analysis of exhaled particles from patients with asthma and healthy controls
}

\author{
A-C. Almstrand*, M. Josefson* ${ }^{\#}$ A. Bredberg*, J. Lausmaa", P. Sjövall", \\ P. Larsson* and A-C. Olin*
}

ABSTRACT: Particles in exhaled air (PEx) may reflect the composition of respiratory tract lining fluid (RTLF); thus, there is a need to assess their potential as sources of biomarkers for respiratory diseases. In the present study, we compared PEx from patients with asthma and controls using time-of-flight-secondary ion mass spectrometry (TOF-SIMS) and multivariate analysis.

Particles were collected using an instrument developed in-house. 15 nonsmoking subjects with physician-diagnosed asthma and 11 nonsmoking healthy controls performed 10 consecutive forced exhalations into the instrument. Particle concentrations were recorded and samples of particles collected on silicon plates were analysed by TOF-SIMS.

Subjects with asthma exhaled significantly lower numbers of particles than controls $(p=0.03)$ and the ratio of unsaturated to saturated phospholipids was significantly lower in samples from subjects with asthma $(0.25$ versus $0.35 ; p=0.036)$. Orthogonal partial least squares-discriminant analysis models showed good separation between both positive and negative spectra. Molecular ions from phosphatidylcholine and phosphatidylglycerol, and protein fragments were found to discriminate the groups.

We conclude that analysis of PEx is a promising method to examine the composition of RTLF. In the present explorative study, we could discriminate between subjects with asthma and healthy controls based on TOF-SIMS spectra from PEx.

KEYWORDS: Asthma, breath test, exhaled biomarkers, particles, phospholipids, surfactant

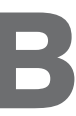

reath analysis is a noninvasive method of gaining information on the respiratory tract and can be useful for monitoring airway disease. Nonvolatile substances in breath are transported in the form of airborne droplets, i.e. particles. Collection of particles in exhaled air (PEx) is a new technique to sample endogenous particles that derive from the respiratory tract. In a previous pilot study, we showed that analysis of PEx using time-of-flight-secondary ion mass spectrometry (TOF-SIMS) provided substantial information on phospholipid composition [1]. Only 10 exhalations are required to detect phospholipids and no sample preparation is needed.

Phospholipids are characteristic components of the surfactant that covers the mucous membranes of the airways [2]. The most abundant lipid is dipalmitoylphosphatidylcholine (PC32:0), which is vital for maintaining a sufficiently low surface tension in the airways. Phosphatidylglycerol (PG), phosphatidylinositol (PI) and sphingomyelin are also present in lower amounts. PG and PI have recently been shown to play important roles in suppressing inflammatory responses $[3,4]$. The phospholipid composition, as measured in bronchoalveolar lavage (BAL), is altered in various respiratory diseases, but the reasons for the changes are uncertain [2]. Thus, analyses of phospholipids in BAL can provide potentially valuable indications of physiological states, but they are far from straightforward, since both the sample preparation and analytical methods involved are laborious and the results may be influenced by large variations in sample recovery and dilution. In addition, BAL is an invasive method associated with certain risks for the patient.

In this study, we investigated and compared TOF-SIMS mass spectra and the composition of
AFFILIATIONS

*Occupational and Environmental Medicine, Sahlgrenska Academy at the University of Gothenburg, Gothenburg,

${ }^{\#}$ Analytical Science, Pharmaceutical Development, AstraZeneca R\&D, Mölndal, and

-SP Technical Research Institute of Sweden, Borås, Sweden.

CORRESPONDENCE

A-C. Almstrand Occupational and Environmental Medicine at Sahlgrenska Academy Box 414 SE-405 30 Gothenburg Sweden E-mail: ann-charlotte.almstrand@ amm.gu.se

Received:

Dec 172010

Accepted after revision:

June 162011

First published online:

June 302011 
phospholipids of PEx from subjects with asthma and controls. TOF-SIMS generates comprehensive spectral data and is therefore attracting increasing interest as a tool for classifying biological samples [5-7]. However, since the method is only semiquantitative and visual interpretation of spectra is difficult, multivariate analysis of the acquired data is often very useful [79]. Thus, multivariate analysis was applied here, using principal component analysis followed by orthogonal partial least squares (OPLS), to screen TOF-SIMS spectra. We hypothesised that, using this approach, TOF-SIMS could be used to discriminate between subjects with asthma and healthy controls, and that the phospholipid composition would differ between the groups.

\section{METHODS}

\section{Study population}

15 nonsmoking subjects (eight females) with physician-diagnosed asthma and 11 nonsmoking healthy controls (six females), who were free from symptoms of respiratory infection for $\geqslant 3$ weeks, participated in the study. They all responded to a respiratory questionnaire based on previous validated items and subjects with asthma were additionally evaluated by a clinical allergist to confirm diagnosis.

Asthma diagnosis was based on the presentation of typical symptoms as defined by Global Initiative for Asthma (GINA) guidelines [10]. A significant measure of variable airway obstruction was documented in 14 out of 15 subjects with asthma as indicated by increased peak expiratory flow variability ( $\geqslant 20 \%$ variation compared with the mean within 1 week), a positive methacholine challenge test (provocative concentration of methacholine using a $20 \%$ fall in forced expiratory volume in $1 \mathrm{~s}(\mathrm{FEV} 1)<8 \mathrm{mg} \cdot \mathrm{mL}^{-1}$ ) or a positive reversibility test (12\% or $200 \mathrm{~mL}$ in FEV1 after $400 \mathrm{mg}$ of inhaled salbutamol or spontaneous variation in FEV1 $(12 \%)$. One of the subjects with asthma was taking high doses of inhaled glucocorticosteroids and long-acting $\beta_{2}$-agonists, had asthma since childhood and was reluctant to taper medication. 10 out of the 15 subjects with asthma were taking inhaled glucocorticosteroids daily and one subject only during the pollen season.

Atopy was defined as a positive skin-prick test to common inhalant allergens.

Control subjects all regarded themselves as healthy and did not report any respiratory symptoms or any medication. All performed a reversibility test with a negative outcome.

The forced vital capacity (FVC) and FEV1 of all participants was determined using a dry-wedge spirometer (Vitalograph ${ }^{\circledR}$; Vitalograph, Maids Moreton, UK). They performed at least three technically acceptable trials in accordance with European Respiratory Society (ERS) guidelines [11], and the largest values for FVC and FEV1 were recorded and compared with predicted values [12]. In addition, the mid-expiratory flow between $25 \%$ and $75 \%$ of FVC (MEF25-75\%) was calculated for all subjects.

The clinical examination started with spirometry. The fraction of exhaled NO ( $F$ eNO) was measured using a handheld device, NIOX mini (Aerocrine, Solna, Sweden). A single-breath test at a flow rate of $50 \mathrm{~mL} \cdot \mathrm{s}^{-1}$ was performed according to American Thoracic Society (ATS)/ERS recommendations [13]. After sampling PEx, skin-prick and reversibility tests were performed.
Methacholine challenge tests were performed on a separate occasion in those subjects where no other objective measure of variable airway obstruction could be obtained.

The study was approved by the Ethics Committee of Sahlgrenska Academy at the University of Gothenburg, Gothenburg, Sweden. Written informed consent was obtained from all participants.

\section{Collection of PEx and TOF-SIMS analysis}

Samples were collected by particle impaction using an instrument developed in-house, as previously described [1]. Participants breathed particle-free air (room air filtered through a HEPA filter; Whatman Inc., NJ, USA) for 3 min before sampling, to avoid contamination from exogenous particles, then performed 10 consecutive forced exhalations, with a target flow of $90 \%( \pm 10 \%)$ of their FEV1, into the device. The flow rate was displayed on a computer screen so that participants could observe and control their breathing. Particle concentrations were recorded with an optical particle counter (Grimm Model 1.108; Grimm Aerosol Technik GmbH \& Co, Ainring, Germany). In addition, PEx were collected on silicon plates that were removed from the instrument immediately after sampling and stored in natural plastic wafer shippers (Fluoroware H22-10; Entegris Inc., Chaska, MN, USA) at $-20^{\circ} \mathrm{C}$, for no longer than 5 months, until analysis. Sample spots were analysed with a TOF-SIMS IV instrument (IONTOF, Münster, Germany) as previously described [1]. Positive- and negative-ion TOF-SIMS spectra were acquired from two randomly selected spots in each sample.

\section{Statistical data analysis}

Univariate analysis based on nonparametric (Mann-Whitney) tests was applied to assess the validity of findings of our pilot study and earlier observations, using SAS 9.1 (SAS Institute Inc., Cary, NC, USA).

\section{Multivariate regression analysis}

For each TOF-SIMS measurement, the spectra from all pixels were summed into a single spectrum, which was binned to a digital resolution of mass $(\mathrm{m}) /$ charge $(z) 0.2$ and converted to Matlab 4 files (Mathworks Inc., Natick, MA, USA) using inhouse software in Python (Python 2.5.2 with NumPy 1.2.1; Python Software Foundation, Wolfeboro Falls, NH, USA). Signal intensities of individual ions, $m / z$ 20-1,000, were normalised according to total intensity in the corresponding full spectrum in Matlab 7.6. SIMCA-P+12.0 (Umetrics AB, Umeå, Sweden) was used for multivariate analysis, after mean-centring and Pareto scaling the data [14, 15].

Principal component analysis (PCA) was used to verify the quality of spectra. OPLS-discriminant analysis (DA) was then used to identify differences between patient groups [16, 17].

PCA is a statistical tool that is used to examine relationships between multiple variables simultaneously, by using latent correlations among them to reduce the dimensions of the data. OPLS-DA is a multivariate method that can be used to identify and display differences between patient groups in a single graph (OPLS-DA loading plot) showing all measured variables. The analysis also produces a score plot showing the separation of the groups based on the content of the loading plot. Q2 is a measure of the quality in multivariate models. For OPLS-DA, Q2 is based on cross-validation, a procedure in 
which fractions of data are systematically excluded. For instance, a model may be calculated using six out of seven of observations (here mass spectra) at a time. A sum of squares is then accumulated for the deviations between the modelled responses (group ratings) and the assigned group (Y). These sum of squares, called the prediction error sum of squares (PRESS), are accumulated for all excluded fractions and compared to the total sum of squares for the variation in $Y$ (SS(Y)) by the formula: Q2=1-PRESS/SS(Y). A value $>0.5$ is considered large and indicates that the model provides useful systematic information for the present type of biological data.

\section{RESULTS}

Characteristics of subjects and the median amounts of PEx collected from them are presented in table 1. Subjects with asthma exhaled significantly lower numbers of PEx (of all size classes) than controls $(\mathrm{p}=0.03)$. The mass median aerodynamic diameter was the same in both groups, i.e. $0.73 \mu \mathrm{m}$.

\section{TOF-SIMS analysis}

The overall patterns of peaks in spectra from subjects with asthma and controls were similar. Typical positive- and negative-ion spectra for a healthy subject are shown in figure 1. In positive-ion spectra, both fragments and molecular ions $\left([\mathrm{M}+\mathrm{H}]^{+},[\mathrm{M}+\mathrm{Na}]^{+}\right.$and $\left.[\mathrm{M}+\mathrm{K}]^{+}\right)$of PC were observed. The major signals were from sodium, potassium and PC fragment ions in the lower mass region, and from various species of PC in the higher mass range. In negative-ion spectra, lower mass regions were dominated by signals from $\mathrm{CN}^{-}, \mathrm{CNO}^{-}$, silicon oxides (e.g. $\mathrm{SiO}_{2}^{-}$and $\left.\mathrm{SiO}_{3} \mathrm{H}^{-}\right), \mathrm{PO}_{3}^{-}$, and the free fatty acids $\mathrm{C} 16: 1, \mathrm{C} 16: 0, \mathrm{C} 18: 1$ and $\mathrm{C} 18: 0$. In higher mass ranges, signals from phosphatidic acid (PA), PG and PI were detected (table 2). Overall, there was high variability in signal intensity among spectra from different individuals. In addition, as observed in our previous study [1], signals from molecular ions were generally stronger in the rim of the spots, probably due to matrix effects.

\section{Multivariate analysis}

The PCA analysis of both positive and negative spectra showed that the final dataset does not have any outliers or groupings depending on uncontrolled factors, e.g. sample handling or instrument instability.
For the OPLS-DA analysis, data from samples from subjects with asthma and controls were placed in two different groups to see if the mass spectra supported this division. The resulting models showed good separation for both positive- and negativeion spectra (see score plots in fig. 2). The corresponding OPLSDA loading plots (figs 3 and 4) show the variation in $\mathrm{m} / \mathrm{z}$ information that explains the division in the score plot. In this context, an asthma patient sample with a positive value on the score $x$-axis corresponds to positive values on the $y$-axis of the loading plot. Peaks contributing to the separation between groups in positive- and negative-ion spectra are visible in the loading plots. In summary, in the low mass range, signals from silicon are stronger among samples from subjects with asthma, while sodium and potassium are stronger among controls. Peaks that are commonly observed in TOF-SIMS spectra, such as phthalates (common contaminants from surrounding air) and fragments from organic compounds, appear strong for samples from both subjects with asthma and controls. In the high mass range, molecular ions from phospholipids (PC30:0+H, PC32:0+H and $\mathrm{PC} 32: 0+\mathrm{Na}$ ) are strong among samples from subjects with asthma. In negative-ion spectra, silicon signals and typical fragments from protein and phosphate are stronger among samples from subjects with asthma. In the high mass range, molecular ions from phospholipids, such as PG28:0, PG32:0, PG34:1 and PG36:1, are higher among samples from subjects with asthma.

In addition, an OPLS-DA analysis was performed solely on positive spectra of samples from subjects with asthma, which were divided into two groups based on the subjects' intake of inhaled glucocorticosteroids. The resulting model showed a complete separation without overlap between subjects taking glucocorticosteroids and subjects not taking glucocorticosteroids (the corresponding score plot is shown in supplementary information). In the higher mass region, signals from molecular ions PC32:0 were stronger in samples from subjects taking glucocorticosteroids.

The within-sample (between-spot) repeatability in the score plot was estimated by calculating the coefficient of variation using the standard deviation of scores in the predictive component divided by the mean distance between subjects with asthma and controls. To test the within-individual variability in the OPLS score plot, double or triple samples

\section{TABLE 1 Basic data on subjects with asthma and controls}

\section{Controls}

\section{Males/females $\mathbf{n}$}

Age yrs

Atopy \%

FVC $\%$ pred

FEV $1 \%$ pred

MEF $25-75 \%$

FeNO ppb

Number of collected particles $0.50-2.0 \mu \mathrm{m}$ median (range)
$5 / 6$

$37(21-55)$

36

$115.3(93-129)$

$108(80-126)$

$3.8(1.6-6.0)$

$20(11-33)$

$44000(4500-2100000)$

Subjects with asthma

$7 / 8$

$30(19-55)$

79

$102.6(77-126)$

$97(75-116)$

$3.6(1.5-6.2)$

$55(13-223)$

$23000(8100-97000)$

Data are presented as mean (range), unless otherwise stated. FVC: forced vital capacity; \% pred: \% predicted; FEV1: forced expiratory volume in 1 s; MEF $25-75 \%$ : midexpiratory flow at $25-75 \%$ of FVC; FeNO: exhaled nitric oxide fraction. ${ }^{*}: n=11 ;{ }^{\bullet}: n=15 ;^{+}$: number based on 14 subjects; data are missing for one subject. 


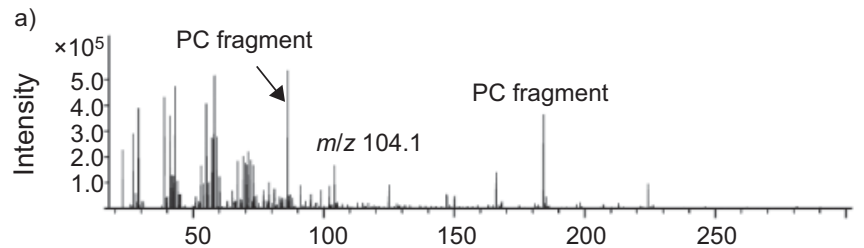

\begin{tabular}{|c|c|c|c|}
\hline TABLE 2 & $\begin{array}{l}\text { lary of } \\
\text { dary ic } \\
\text { ed fro }\end{array}$ & $\begin{array}{l}\text { ssignments } \\
\text { s spectrome } \\
\text { led particles }\end{array}$ & $\begin{array}{l}\text {-of-flight- } \\
\text { ctra }\end{array}$ \\
\hline \multicolumn{2}{|c|}{ Positive ions } & \multicolumn{2}{|c|}{ Negative ions } \\
\hline Assignment & $m / z$ & Assignment & $m / z$ \\
\hline PC fragment & 58.1 & C16:1 & 253.2 \\
\hline PC fragment & 86.1 & C16:0 & 255.2 \\
\hline PC fragment & 184.1 & C18:1 & 281.2 \\
\hline PC fragment & 476.4 & C18:0 & 283.3 \\
\hline PC fragment & 478.4 & PA32:1 & 645.4 \\
\hline $\begin{array}{c}\text { Lyso-PC/PC } \\
\text { fragment }\end{array}$ & 494.3 & PA32:0 & 647.4 \\
\hline $\begin{array}{c}\text { Lyso-PC/PC } \\
\text { fragment }\end{array}$ & 522.3 & PG28:1 & 663.4 \\
\hline $\begin{array}{c}\text { Lyso-PC/PC } \\
\text { fragment }\end{array}$ & 524.4 & PG28:0 & 665.4 \\
\hline PC28:0+H & 678.5 & PG32:0 & 671.5 \\
\hline РС30:0+H & 706.6 & PA34:1 & 673.5 \\
\hline PC30:0+Na & 728.5 & PG32:0 & 721.5 \\
\hline PC32:1+H & 732.6 & PG34:1 & 747.5 \\
\hline PC32:0+H & 734.5 & PG36:2 & 773.5 \\
\hline РС30:0+K & 744.5 & PG36:1 & 775.5 \\
\hline $\mathrm{PC} 32: 1+\mathrm{Na}$ & 754.5 & $\mathrm{PI} 34: 2$ & 833.6 \\
\hline $\mathrm{PC} 32: 0+\mathrm{Na}$ & 756.5 & PI34:1 & 835.6 \\
\hline PC34:1+H & 760.6 & PI36:2 & 861.6 \\
\hline PC34:0+H & 762.6 & PI36:1 & 863.6 \\
\hline PC32:1+K & 770.6 & & \\
\hline РС32:0+K & 772.5 & & \\
\hline $\mathrm{PC} 34: 1+\mathrm{Na}$ & 782.5 & & \\
\hline PC34:0+Na & 784.5 & & \\
\hline PC34:1+K & 798.6 & & \\
\hline PC34:0+K & 800.6 & & \\
\hline
\end{tabular}

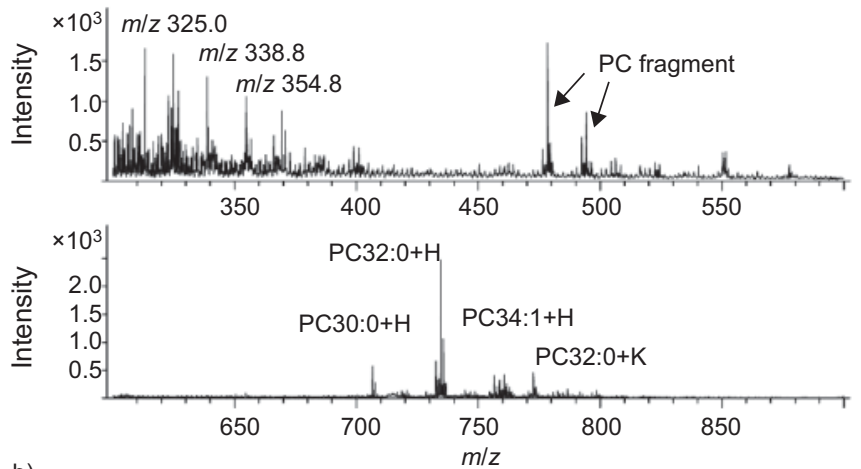

b)
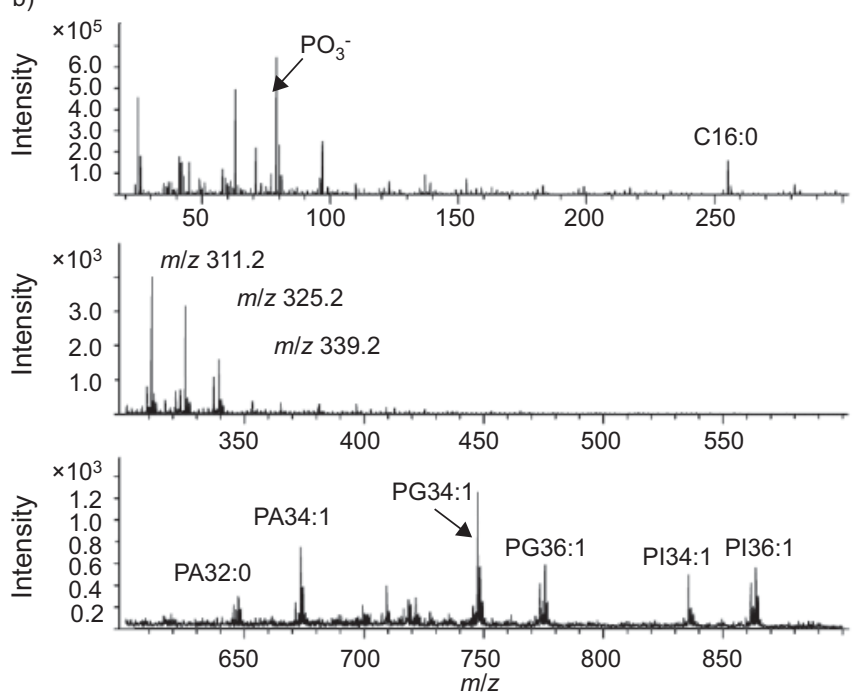

All mass $(m) /$ charge $(z)$ values correspond to singly charged monoisotopic ions. Molecular species of phospholipids are named $x: a$, where $x$ is the number of carbons and $a$ is the number of double bonds. Secondary ions are detected as protonated ions $[\mathrm{M}+\mathrm{H}]^{+}$, sodiated ions $[\mathrm{M}+\mathrm{Na}]^{+}$and potassiated ions $[\mathrm{M}+\mathrm{K}]^{+}$in positive ionisation mode and deprotonated $[\mathrm{M}-\mathrm{H}]^{-}$in negative ionisation mode. PC: phosphatidylcholine; C: free fatty acid; PA: phosphatidic acid; PG: phosphatidylglycerol; PI: phosphatidylinositol.

healthy control. b) A typical negative-ion TOF-SIMS mass spectrum from a sample spo of exhaled particles from a healthy control. PC: phosphatidylcholine; PA: phosphatidic acid; PG: phosphatidylglycerol; PI: phosphatidylinositol; $m$ : mass; $z$ : charge.

from three controls and one subject with asthma were included in the original model which then was re-fitted. The coefficient of variation within-sample was $14 \%$ and within-individual $17 \%$. For ratios of unsaturated to saturated phospholipids, the coefficient of variation within-samples was $4 \%$ and withinindividual was $26 \%$.

\section{Study of possible matrix effects}

As the controls exhaled significantly higher amounts of PEx than subjects with asthma, the possible effects of variations in sample amounts on the spectra were assessed by a separate OPLS analysis, using solely spectra from control samples, in which particle concentrations were the $\mathrm{Y}$ variables and the TOF-SIMS spectra the $X$ variables. A strong correlation was found between particle concentration and variation in molecular ions of phospholipids (Q2=0.97). PC32:0+H, PC32:0+Na

and $\mathrm{PC} 32: 0+\mathrm{K}$ (and in the low mass range $\mathrm{Si}, \mathrm{K}, \mathrm{SiOH}$ and phthalate fragments) were stronger in positive-ion spectra of samples with low particle amounts. PG34:1 and PG36:1 were the identified phospholipids that dominated in negative-ion spectra of samples with low particle amounts.

\section{Univariate analysis}

We also tested the validity of findings from our previous pilot study by applying univariate analysis to data acquired in the present study. The results indicated, contrary to previous findings, that ratios of levels of $\mathrm{PO}_{3}^{-}$to $\mathrm{CN}^{-}, \mathrm{CNO}^{-}$and the sum of $\mathrm{CN}^{-}$ and $\mathrm{CNO}^{-}$in PEx did not significantly differ between the groups.

The ratio of unsaturated to saturated phospholipids was significantly lower in samples from subjects with asthma than in samples from controls ( 0.25 versus $0.35 ; \mathrm{p}=0.036)$, despite 

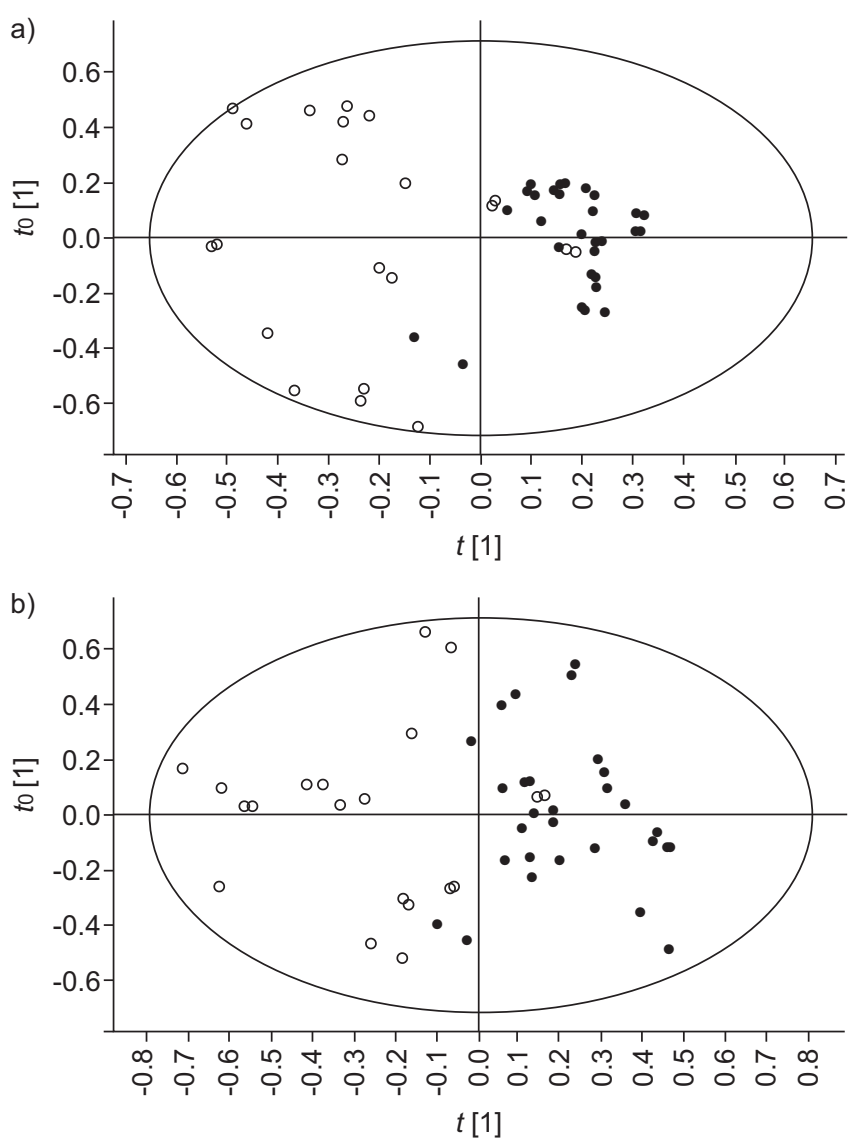

FIGURE 2. Orthogonal partial least squares-discriminant analysis score plot of a) positive-ion spectra $(\operatorname{R} 2 X(\mathrm{cum})=0.606, \mathrm{R} 2 \mathrm{Y}$ (cum) $=0.655, \mathrm{Q} 2$ (cum) $=0.517$, where $\mathrm{R} 2 \mathrm{X}$ (cum) is the cumulative sum of squares (SS) of all the $X$ values explained by all extracted components, $R 2 Y$ (cum) is the cumulative $S S$ of the $Y$ values explained by all extracted components, and Q2(cum) is the cumulative Q2 for the extracted components), and b) negative-ion spectra $(R 2 X($ cum $)=0.694, R 2 Y($ cum $)=0.602$, $Q 2($ cum $)=0.568$ ). Duplicate samples were analysed. $t$ [1]: the predictive component; $t_{0}$ [1]: first orthogonal component. $\bigcirc$ : subjects with asthma; $\bullet$ : control.

high variability within each group (0.13-0.42 and 0.14-0.68, respectively).

\section{DISCUSSION}

Sampling and analysing PEx, which have phospholipid profiles indicating an origin in RTLF [1], offer a novel, noninvasive way of examining the physiological status of subjects' airways. In the present study, we discriminated between subjects with asthma and healthy controls using multivariate analysis of TOF-SIMS spectra of PEx. Loading plots from the OPLS analysis show that fragment ions and inorganic ions (present in the low mass range) are important differentiators between the groups. It is difficult to interpret the variation in individual fragment peaks, although their pattern is significant for the division of the groups. Hence, we focus on the molecular ions to address possible reasons for the observed differences in the mass spectra. Major differences in this respect were that spectra of samples from subjects with asthma yielded stronger signals of PC32:0 and several PG species compared with controls.
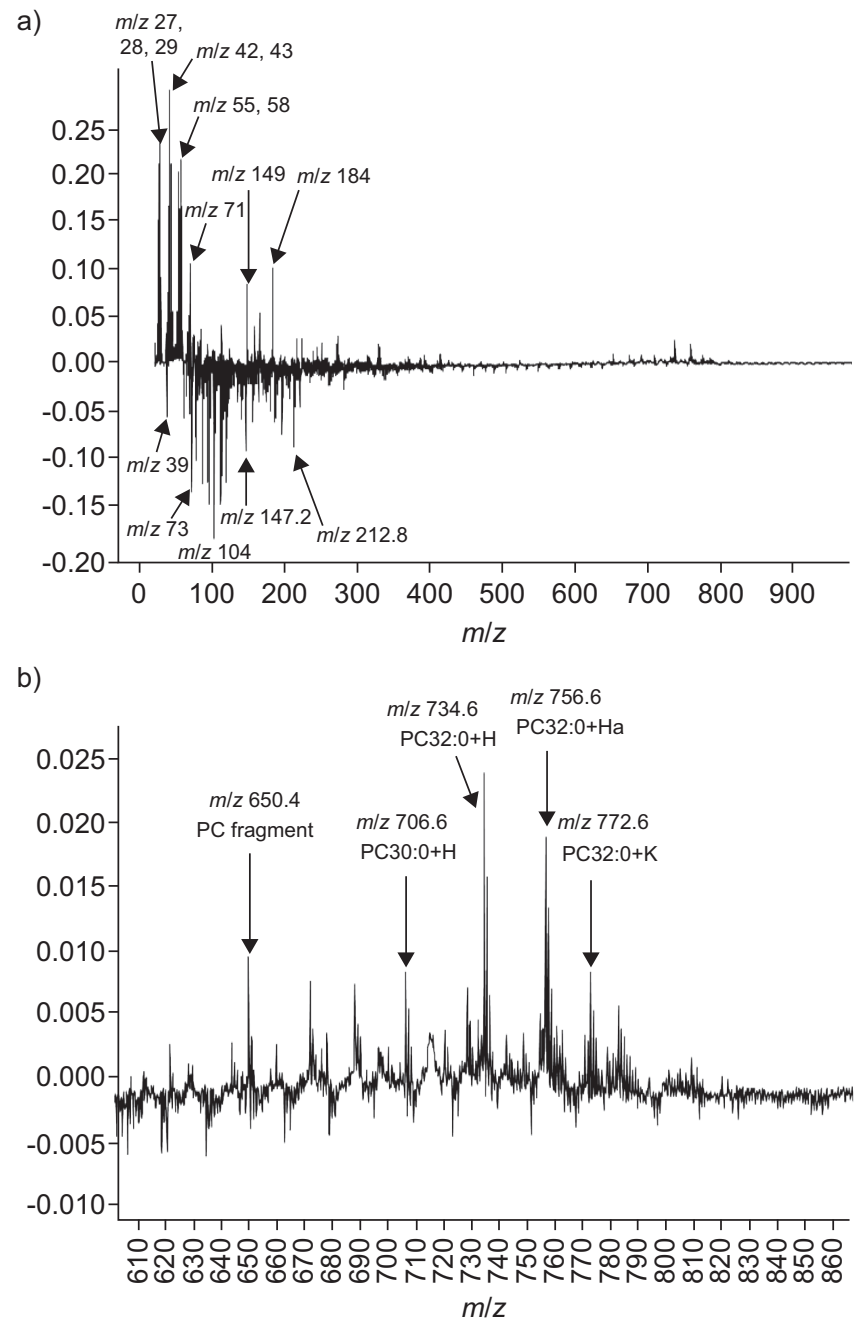

FIGURE 3. a) Orthogonal partial least squares-discriminant analysis (OPLSDA) loading plot of positive-ion time-of-flight-secondary ion mass spectrometry (TOF-SIMS) spectra. Arrows indicate the $\mathrm{m} / \mathrm{z}$ peaks with strong impacts on the separation between groups. A positive value on the $y$-axis corresponds to $\mathrm{m} / \mathrm{z}$ peaks that are relatively stronger in spectra of samples from subjects with asthma than in control spectra. b) Detailed view of the high mass range of the OPLS-DA loading plot (shown in entirety in part a) of positive-ion TOF-SIMS spectra. Identified peaks are indicated. PC: phosphatidylcholine; $m$ : mass; $z$ : charge.

Alterations in the phospholipid composition in RTLF associated with asthma have been rarely studied; previous studies of associated changes in the composition of airways surfactant have focused on induced sputum or BAL. It has been speculated that in asthma, surfactant dysfunction (due to decreased surface activity) is involved in airway obstruction and inflammation [18-20]. WRIGHT et al. [21] observed that the proportion of PC32:0 was decreased in sputum, but not in BAL in patients with asthma, while CHANG et al. [22] found that PC32:0 correlated with eosinophilic cationic protein in sputum of children with asthma. It has also been observed that children with asthma have higher levels of PC32:0 in sputum compared with control subjects [23]. In atopic asthma, experimental antigen exposure has been shown to induce a reduction in PG in large surfactant aggregates collected from BAL [24]. Leakage of plasma proteins seems to be important for inactivation of 


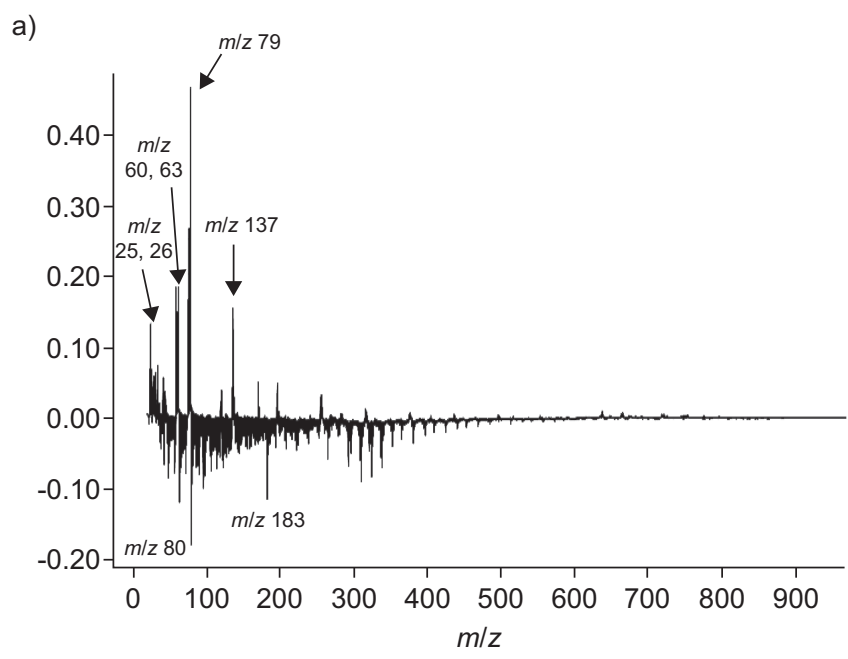

b)

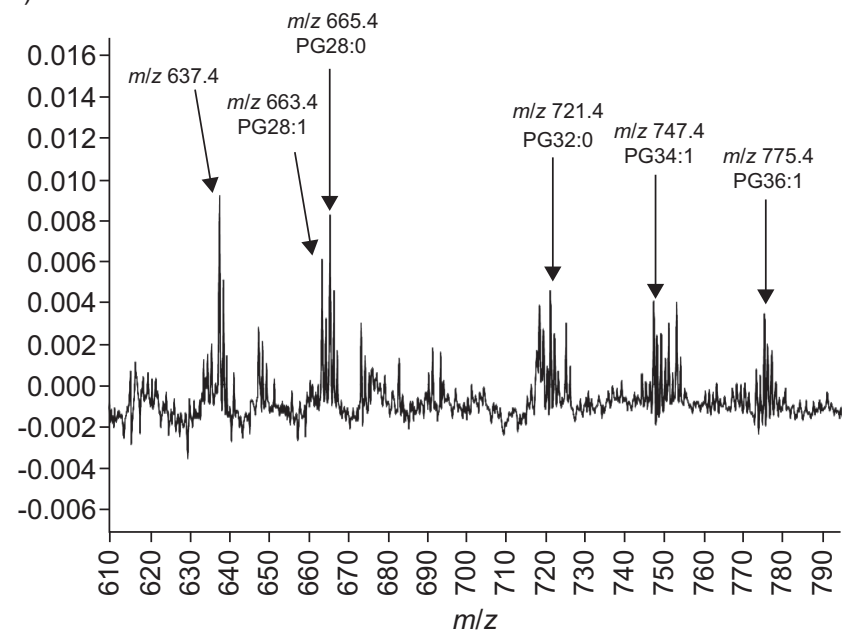

FIGURE 4. a) Orthogonal partial least squares-discriminant analysis (OPLSDA) loading plot of time-of-flight-secondary ion mass spectrometry (TOF-SIMS) negative-ion spectra. Arrows indicate the mass $(m) /$ charge $(z)$ peaks with strong impacts on the separation between groups. A positive value on the $y$-axis corresponds to $\mathrm{m} / \mathrm{z}$ peaks that are relatively stronger in spectra of samples from subjects with asthma than in control spectra. b) Detailed view of the high mass range of the OPLS-DA loading plot (shown in entirety in part a) of TOF-SIMS negative-ion spectra. Identified peaks are indicated. PG: phosphatidylglycerol.

surfactant [19, 21, 25]. It is not possible to identify specific proteins by TOF-SIMS, but $\mathrm{CN}^{-}$and $\mathrm{CNO}^{-}$are considered unspecific fragments from proteins and peptides, and according to the loading plots, both $\mathrm{CN}^{-}$and $\mathrm{CNO}^{-}$were elevated among subjects with asthma. The ratio of proteins to phospholipids can be estimated by comparing signals from $\mathrm{CN}^{-}$and $\mathrm{CNO}^{-}$, and the signal from $\mathrm{PO}_{3}^{-}$, which is a strong phospholipid fragment. In the present study, we observed no significant difference in this ratio between groups. We also investigated the difference in the ratio of saturated to unsaturated phospholipids between the groups and found that the ratio was significantly elevated among asthmatics. Unsaturated fatty acids are more rapidly oxidised than saturated species, suggesting that an increased ratio of saturated to unsaturated species may reflect the increased oxidative stress known to occur in asthma [26].
In both negative- and positive-ion spectra, molecular ions from phospholipids were stronger in spectra from subjects with asthma. Indeed, no phospholipid molecular ions were stronger in spectra of samples from controls. This distinction may be due to samples from asthmatic subjects containing smaller amounts of particles. The possible matrix effects caused by variations in sample amounts were therefore investigated further. Some of the phospholipid molecular peaks were found to correlate to the particle amount. In positive spectra, low particle amounts were correlated with stronger signals from PC32:0, while in negative-ion spectra, low particle amounts correlated with stronger signals from PG34:1 and PG36:1. However, PG28:0 did not correlate with particle amounts, but is important for distinguishing between controls and subjects with asthma. Samples from two control subjects had extremely high particle concentrations (400,000 and 2,100,000 particles, respectively; see table 1 for median values), and, since particle concentration was shown to affect the chemical analysis, the investigated relationships were also modelled without data from these subjects. The Q2 values for the resulting models of both negative- and positive-ion spectra were improved $(0.52$ versus 0.91 for positive spectra and 0.57 versus 0.64 for negative spectra) and the OPLS loadings for the models for asthma and the model for particle amount were not correlated. The division between groups in the new models was stronger than in the original models (see online supplementary material for score plots). Furthermore, PC32:0, PG32:0, PG34:1 and PG36:1 were stronger differentiators of the groups in the new models, indicating that the method could be further improved by standardising sampled particle numbers for TOF-SIMS analysis.

The effect of inhaled glucocorticosteroids on spectra from subjects with asthma was also examined using OPLS-DA. The results indicate that inhaled glucocortisteroids may alter the surfactant composition and, hence, the spectra of PEx. The results agree with studies showing that glucocorticosteroids stimulate the synthesis of PC [27, 28]. An alternative explanation is that those taking inhaled glucocortisteroids have a more severe disease and that this is reflected in the mass spectra. The groups were, however, too small to draw any further conclusions.

It is not known if the number of PEx is affected in airway inflammation. In the present study, controls exhaled significantly higher numbers of PEx than subjects with asthma. Subjects performed forced exhalations, since this manoeuvre produces much higher particle numbers than tidal exhalations. The subjects were instructed to exhale at a maximal flow corresponding to $90 \%$ of their FEV1 $( \pm 10 \%)$. The reasoning behind this was that the intra-thoracic flow may be highly important for the formation of particles during forced exhalations, especially during dynamic compression of the airways, which may be an important mechanism for endogenous particle production. In this small group, inhaled glucocorticosteroids did not affect particle number. We have recently shown that airway re-opening is an important mechanism for PEx formation [29]. We have not controlled for this in the present study. However, all subjects were given similar instructions: to inhale as deeply as possible and then exhale maximally. Subjects with asthma may, however, have been unable to exhale as deeply as control subjects because of premature airway closure. The site of airway closure may have taken place at earlier generations of the bronchial tree for subjects with asthma compared with controls, which could affect the 
particle number and possibly also the composition of particles. Furthermore, the location of the flow-limiting segment may differ between subjects with asthma and controls, which in turn may also affect the location of particle production if particles are formed due to shear forces produced by the high flows.

Differences in the composition of the RTLF may also contribute to the observed difference in PEx concentrations between controls and subjects with asthma. Nebulised isotonic saline has been shown to influence the number of PEx during tidal breathing [30], possibly by influencing the viscosity of the respiratory tract lining fluid. Whether PEx concentrations are really lowered in asthma is a matter for future studies.

Asthma is a very heterogeneous disease; ideally more subjects would have been included in our study, and possibly steroidnaïve subjects with a more uniform disease. The subjects with asthma in the present study were recruited by advertisements in the hospital or the university and presented with varying disease severity (from mild-intermittent to moderate) and medication varied. Hence, the present work should be considered an explorative study of both a new sampling method and an analytical method that has not previously been used in respiratory research. We were nevertheless able to show that it is possible to distinguish between subjects with asthma and controls based on TOF-SIMS spectra of PEx and that differences in the phospholipid composition were important for the separation between groups.

\section{Conclusions}

Collection and analysis of PEx seem to be a promising new method for examining the RTLF and monitoring changes in the composition of surfactant in respiratory disease. The signals from PC32:0 and several species of PG were higher in samples from asthmatic subjects, and these subjects also had higher ratios of saturated to unsaturated phospholipids compared with controls.

\section{SUPPORT STATEMENT}

This study was sponsored by the Swedish Heart-Lung Foundation.

\section{STATEMENT OF INTEREST}

Statements of interest for A-C. Almstrand, M. Josefson, J. Lausmaa and A-C. Olin can be found at www.erj.ersjournals.com/site/misc/ statements.xhtml

\section{ACKNOWLEDGEMENTS}

We thank A. Claesson (Occupational and Environmental Medicine, Sahlgrenska Academy at University of Gothenburg, Gothenburg, Sweden) for help with spirometry, skin-prick tests and reversibility tests, and E. Mirgorodskaya, K. Torén (Occupational and Environmental Medicine, Sahlgrenska Academy at University of Gothenburg) and B. Bake (Respiratory Medicine and Allergology, Sahlgrenska Academy at University of Gothenburg) for valuable comments on the manuscript. We also thank all study participants.

\section{REFERENCES}

1 Almstrand AC, Ljungstrom E, Lausmaa J, et al. Airway monitoring by collection and mass spectrometric analysis of exhaled particles. Anal Chem 2009; 81: 662-668.

2 Griese M. Pulmonary surfactant in health and human lung diseases: state of the art. Eur Respir J 1999; 13: 1455-1476.

3 Kuronuma K, Mitsuzawa H, Takeda K, et al. Anionic pulmonary surfactant phospholipids inhibit inflammatory responses from alveolar macrophages and u937 cells by binding the lipopolysaccharide-interacting proteins cd14 and md-2. J Biol Chem 2009; 284: 25488-25500.

4 Numata M, Chu HW, Dakhama A, et al. Pulmonary surfactant phosphatidylglycerol inhibits respiratory syncytial virus-induced inflammation and infection. Proc Natl Acad Sci USA 2010; 107: 320-325.

5 Sjovall P, Lausmaa J, Johansson B. Mass spectrometric imaging of lipids in brain tissue. Anal Chem 2004; 76: 4271-4278.

6 Brunelle A, Laprevote O. Lipid imaging with cluster time-of-flight secondary ion mass spectrometry. Anal Bioanal Chem 2009; 393: 31-35.

$7 \mathrm{Wu}$ L, Lu X, Kulp KS, et al. Imaging and differentiation of mouse embryo tissues by TOF-SIMS. Int J Mass Spectrom 2007; 260: 137-145.

8 Park J-W, Min H, Kim Y-P, et al. Multivariate analysis of TOFSIMS data for biological applications. Surf Interface Anal 2009; 41: 694-703.

9 Lhoest JB, Wagner MS, Tidwell CD, et al. Characterization of adsorbed protein films by time of flight secondary ion mass spectrometry. J Biomed Mater Res 2001; 57: 432-440.

10 Global Initiative for Asthma. Pocket guide for asthma management and prevention. www.ginasthma.org Date last accessed: September 16, 2011.

11 Miller MR, Hankinson J, Brusasco V, et al. Standardisation of spirometry. Eur Respir J 2005; 26: 319-338.

12 Quanjer PH, Tammeling GJ, Cotes JE, et al. Lung volumes and forced ventilatory flows. Report working party standardization of lung function tests, European Community for steel and coal. Official statement of the European Respiratory Society. Eur Respir J 1993; 16: Suppl. 6, 5-40.

13 American Thoracic Society, European Respiratory Society. ATS/ ERS recommendations for standardized procedures for the online and offline measurement of exhaled lower respiratory nitric oxide and nasal nitric oxide. Am J Respir Crit Care Med 2005; 171: 912-930.

14 Trygg J, Holmes E, Lundstedt T. Chemometrics in metabonomics. J Proteome Res 2007; 6: 469-479.

15 van den Berge M, ten Hacken NHT, Cohen J, et al. Small airway disease in asthma and COPD. Chest 2011; 139: 412-423.

16 Trygg J, Wold S. Orthogonal projections to latent structures (OPLS). J Chemometrics 2002; 16: 119-128.

17 Wold S, Trygg J, Berglund A, et al. Some recent developments in PLS modeling. Chemometrics Intelligent Lab Sys 2001; 58: 131-150.

18 Kurashima K, Fujimura M, Matsuda T, et al. Surface activity of sputum from acute asthmatic patients. Am J Respir Crit Care Med 1997; 155: 1254-1259.

19 Hohlfeld JM, Ahlf K, Enhorning G, et al. Dysfunction of pulmonary surfactant in asthmatics after segmental allergen challenge. Am J Respir Crit Care Med 1999; 159: 1803-1809.

20 Jarjour NN, Enhorning G. Antigen-induced airway inflammation in atopic subjects generates dysfunction of pulmonary surfactant. Am J Respir Crit Care Med 1999; 160: 336-341.

21 Wright SM, Hockey PM, Enhorning G, et al. Altered airway surfactant phospholipid composition and reduced lung function in asthma. J Appl Physiol 2000; 89: 1283-1292.

22 Chang AB, Gibson PG, Masters IB, et al. The relationship between inflammation and dipalmitoyl phosphatidycholine in induced sputum of children with asthma. J Asthma 2003; 40: 63-70.

23 Shaheen MA, Mahmoud MA, Abdel Aziz MM, et al. Sputum dipalmitoylphosphatidylcholine level as a novel airway inflammatory marker in asthmatic children. Clin Respir J 2009; 3: 95-101.

24 Hite RD, Seeds MC, Bowton DL, et al. Surfactant phospholipid changes after antigen challenge: a role for phosphatidylglycerol in dysfunction. Am J Physiol Lung Cell Mol Physiol 2005; 288: L610-L617.

25 Heeley EL, Hohlfeld JM, Krug N, et al. Phospholipid molecular species of bronchoalveolar lavage fluid after local allergen 
challenge in asthma. Am J Physiol Lung Cell Mol Physiol 2000; 278: L305-L311.

26 Ochs-Balcom HM, Grant BJ, Muti P, et al. Antioxidants, oxidative stress, and pulmonary function in individuals diagnosed with asthma or COPD. Eur J Clin Nutr 2006; 60: 991-999.

27 Post M, Batenburg JJ, van Golde LM. Effects of cortisol and thyroxine on phosphatidylcholine and phosphatidylglycerol synthesis by adult rat lung alveolar type II cells in primary culture. Biochim Biophys Acta 1980; 618: 308-317.
28 Bunt JE, Carnielli VP, Janssen DJ, et al. Treatment with exogenous surfactant stimulates endogenous surfactant synthesis in premature infants with respiratory distress syndrome. Crit Care Med 2000; 28: 3383-3388.

29 Almstrand AC, Bake B, Ljungstrom E, et al. Effect of airway opening on production of exhaled particles. J Appl Physiol 2010; 108: 584-588.

30 Edwards DA, Man JC, Brand P, et al. Inhaling to mitigate exhaled bioaerosols. Proc Natl Acad Sci USA 2004; 101: 17383-17388. 